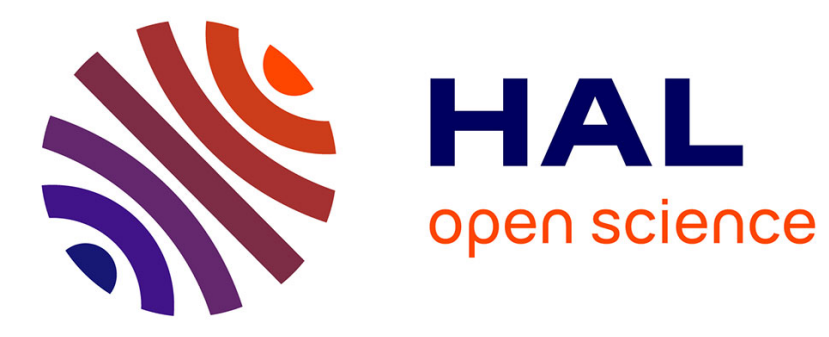

\title{
L'incontinence coïtale
}

M. Moutounaïck, G. Miget, M. Teng, F. Kervinio, C. Chesnel, A. Charlanes, F. Le Breton, Gérard Amarenco

\section{To cite this version:}

M. Moutounaïck, G. Miget, M. Teng, F. Kervinio, C. Chesnel, et al.. L'incontinence coïtale. Progrès en Urologie, 2018, 28 (11), pp.515-522. 10.1016/j.purol.2018.05.001 . hal-01989946

\section{HAL Id: hal-01989946 https://hal.sorbonne-universite.fr/hal-01989946}

Submitted on 22 Jan 2019

HAL is a multi-disciplinary open access archive for the deposit and dissemination of scientific research documents, whether they are published or not. The documents may come from teaching and research institutions in France or abroad, or from public or private research centers.
L'archive ouverte pluridisciplinaire HAL, est destinée au dépôt et à la diffusion de documents scientifiques de niveau recherche, publiés ou non, émanant des établissements d'enseignement et de recherche français ou étrangers, des laboratoires publics ou privés. 


\section{L’incontinence coütale}

MOUTOUNAÏCK M., MIGET G, TENG M., KERVINIO F., CHESNEL C., CHARLANES A., LE BRETON F., AMARENCO G.

Sorbonne Université, GRC 01, GREEN - Groupe de Recherche Clinique en Neuro-Urologie, AP-HP, Hôpital Tenon, 75020 Paris, France

\section{Résumé}

Introduction: Au-delà du retentissement de l'incontinence urinaire sur la qualité de vie, l'image de soi et indirectement sur la vie sexuelle, des fuites urinaires peuvent électivement survenir au cours des rapports sexuels, définissant ainsi l'incontinence coïtale, et pouvant altérer spécifiquement la vie sexuelle.

Objectif : Analyser la prévalence, les mécanismes physiopathologiques et les possibles options thérapeutiques de l'incontinence urinaire coïtale.

Matériels et méthodes: Nous avons effectué une recherche PubMed des termes liés à l'incontinence urinaire et à la dysfonction sexuelle.

Résultats: L'incontinence coïtale était un symptôme peu rapporté de façon spontanée alors qu'elle était présente chez au moins 1/4 des femmes incontinentes. Certaines femmes n'avaient qu'une incontinence coïtale (7,6 à 20\% des cas). Chez l'homme, l'incontinence urinaire pendant les rapports sexuels s'observait principalement après prostatectomie dans 20 à $64 \%$ des cas. Cette incontinence nécessite une analyse sémiologique fine car elle peut survenir à différentes phases du coït (stimulation locale 20-30\%, excitation 13-18\%, pénétration 62,9-68\%, mouvements de va et vient, orgasme $27-37,1 \%$ ) et correspondre ainsi à des mécanismes physiopathologiques différents (hypermobilité cervico-urétrale, incompétence sphinctérienne, instabilité urétrale, hyperactivité détrusorienne). Chez l'homme, l'étiopathogénie la plus fréquente était une incontinence d'effort par insuffisance sphinctérienne. Des thérapeutiques spécifiques ont montré leur efficacité. La rééducation périnéale ( $\mathrm{RR}=0.25$, IC [0.06-1.01]), les thérapeutiques médicamenteuses (anticholinergiques efficaces dans 59\% des cas) ou chirurgicales (bandelettes avec une efficacité de $87 \%$ ) ont été proposées aux patients.

Conclusion: L'incontinence coïtale est un symptôme fréquent et gênant. Son évaluation permet une présomption physiopathologique et une adaptation des stratégies thérapeutiques.

Mots clés: « coital incontinence » ; « urine leakage during sexual activity »; « urinary incontinence »; « sexual dysfunction »

\section{Abstract}

Introduction: Urinary incontinence may seriously impact quality of life, self-image and subsequently the sexual life. Beside this fact, urinary leakage can specifically occur during sexual intercourse, formally named coital incontinence, and thus lead to specific alteration of the sexual life.

Aim: To analyse the prevalence, pathophysiological mechanisms and possible therapeutic options for coital urinary incontinence. 
Methods: Related terms to urinary incontinence and sexual dysfunction were search on PubMed database.

Results: Whereas at least a quarter of incontinent women have a coital incontinence, this symptom was rarely spontaneously reported. Some women had only coital incontinence $(7,6$ to $20 \%$ of cases). In men, urinary incontinence during sexual intercourse was mainly observed after prostatectomy in 20 to $64 \%$ of cases. Coital incontinence requires precise assessment. Indeed, it can occur whatever the phase of coitus : local stimulation (20-30\%), excitement (13-18\%), penetration (62,9-68\%), movements back and forth, orgasm (27-37,1\%). Cervicourethral hypermobility, sphincter incompetence, urethral instability, detrusor overactivity could be the principal physiopathological mechanisms. In men, the main cause was a stress incontinence secondary to sphincter deficiency. Specific therapeutic strategies have proved their effectiveness. The rehabilitative approach ( $\mathrm{RR}=0.25$, IC [0.06-1.01]), medicinal (anticholinergic were effective in 59\% of cases) or surgical therapeutic (slings with an efficiency of $87 \%$ ) was proposed to patients.

Conclusion: Coital incontinence is a common and troublesome symptom. Its precise assessment may suggest a specific mechanism and thus a specific treatment.

Keywords: « coital incontinence »; « urine leakage during sexual activity »; « urinary incontinence »; « sexual dysfunction »

\section{INTRODUCTION}

L'incontinence urinaire détermine très habituellement une altération de la qualité de vie, une gêne sociale et un retentissement psychologique chez les femmes qui en souffrent. Ce retentissement global peut interférer avec la vie sexuelle et les relations de couple par l'image négative renvoyée par l'incontinence tant en terme d'autoconsidération ( «image de soi ») que de représentation symbolique vécue par le partenaire (perte d'attractivité réciproque). Les patients incontinents font ainsi face à des troubles de la fonction sexuelle(1) tels qu'une baisse de la libido, une hyporgasmie, une diminution de la lubrification vaginale, ce qui peut entrainer un évitement des rapports sexuels par les patients ou leur partenaire. Au-delà de ce retentissement général, des fuites urinaires peuvent survenir électivement pendant les rapports sexuels, créant encore d'autres raisons d'altération de la vie sexuelle. D'après la définition traduite de la société internationale de la continence, «l'incontinence percoïtale est une perte involontaire d'urines lors du coït, dont se plaint la patiente. Une définition plus précise distingue la perte d'urines survenant à la pénétration et celle se produisant lors de l'orgasme $\gg(2)$.

L'objectif de cette revue de la littérature était d'analyser la prévalence, les mécanismes physiopathologiques et les possibles options thérapeutiques de l'incontinence urinaire coïtale.

\section{MATERIELS ET METHODES}

Nous avons effectué une recherche sur Pubmed en utilisant les mots clés suivants : « urinary incontinence », «coitus », « coital incontinence », « urine leakage during sexual activity », «female ejaculation », « squirting », « orgasmic incontinence », « sexual dysfunction », «female sexual function », «prostatectomy », « climacturia », « sexual incontinence », « foreplay incontinence », « quality of life », « sexual questionnaires », «neurological conditions », « detrusor overactivity », « stress urinary incontinence », « anticholinergic agents », « giggle incontinence », « laughter ». 
Les articles, écrits en français et en anglais, ont été sélectionnés grâce à leur titre et au résumé. La sélection finale a été faite après lecture entière de l'article.

\section{RESULTATS}

\section{Chez la femme}

L'incontinence coïtale était un symptôme peu rapporté de façon spontanée(3) alors qu'elle était présente chez au moins un quart des femmes incontinentes. Selon les séries(4), 24 à $66 \%$ des femmes incontinentes souffriraient d'incontinence coïtale. Elle est définie par l'existence d'une fuite urinaire pendant le rapport sexuel quelqu'en soit le moment et peut ainsi survenir à toutes les phases du coït. On différenciait alors les fuites urinaires survenant au cours des préliminaires notamment lors de la stimulation locale (20-30\%), l'excitation (13-18\%), celles survenant au moment de la pénétration (62,9 à 68\%), celles pendant les mouvements de va et vient et enfin celles au moment de l'orgasme (27\% à 37\%)(5-7). Cette incontinence pouvait être associée ou non à d'autre type d'incontinence. La prévalence de la symptomatologie pure était de 7,6 à 20\%(5,8,9). Bien qu'il n'existait pas de score spécifique l'évaluant(6), l'utilisation d'une échelle d'incontinence urinaire notamment de qualité de vie (POP-Q, PISQ-12, ICIQ-SF, QoL, KHQ)(7) associée à une échelle de la fonction sexuelle (FSFI, IIEF) était recommandée.

L'incontinence coïtale est multifactorielle. Parmi les facteurs de risque significatifs qui ont été décrit, apparaissait la multiparité(5), la présence de prolapsus(10), l'obésité (OR $=2,47$, IC $[1,13-4,32], \mathrm{p}=0,027)$, la diminution de la pression urétrale maximale de clôture $(\mathrm{OR}=4,56$, IC [1,51-13,59], $\mathrm{p}=0,007)(8)$, la consommation de tabac (OR = 1,4, IC [1,26-1,65], $\mathrm{p}<$ $0,001)$, la prise d'antidépresseurs $(\mathrm{OR}=1,4$, IC $[1,17-1,66], \mathrm{p}<0,001)(9)$. Il s'agit relativement des mêmes facteurs de risque que l'incontinence urinaire classique à l'effort ou sur impériosité. A l'exception de la ménopause et des antécédents de chirurgie pelvienne qui ne prédisposaient pas à une incontinence coïtale.

En 1988 Hilton(3) a proposé une classification des fuites urinaires per-coïtales en distinguant celles qui survenaient avec l'excitation ou pendant les préliminaires, celles pendant la pénétration et/ou pendant l'orgasme. Il a décrit une association avec des diagnostics urodynamiques. Parmi les femmes ayant une incontinence urinaire à l'effort $70 \%$ présentaient une incontinence à la pénétration et $42 \%$ pendant l'orgasme. Parmi celles ayant une hyperactivité détrusorienne, $2 \%$ avaient des fuites pendant la pénétration et $34 \%$ pendant l'orgasme. Dans une étude prospective, Serati et al(11) ont montré une association entre l'hyperactivité détrusorienne et l'incontinence pendant l'orgasme. En effet, les femmes incontinentes pendant l'orgasme avaient une hyperactivité détrusorienne dans $69 \%$ des cas en comparaison avec les femmes incontinentes pendant la pénétration $(29 \%, p<0.0001)$. Dans une étude prospective(7), ils ont également montré que ce premier groupe de femmes avait, à l'échographie, une paroi de vessie significativement plus épaisse que celle ayant une hyperactivité détrusorienne isolée ( $\mathrm{p}=0.007$ ). Ils ont ainsi conclu que l'incontinence pendant l'orgasme était un marqueur de sévérité de l'hyperactivité détrusorienne. Dans une étude incluant 196 femmes incontinentes sexuellement actives dont 66 (34\%) se plaignant d'incontinence coïtale, Vierhout et Gianotten(12) ont réparti les causes de fuites coïtales en facteurs mécaniques et non mécaniques. Ceux considérés comme responsables de fuites urinaires étaient la pression exercée sur l'abdomen et la pénétration profonde (77\%), l'orgasme (74\%) et la stimulation du clitoris (50\%). Khan et al(13) ont concentré leur attention sur l'incontinence survenant au moment de l'orgasme. Ils ont réalisé une étude, incluant 3 femmes, ayant pour but d'évaluer les effets de l'orgasme sur les pressions du détrusor et de 
l'urètre. Pour cela, ils ont effectué un bilan urodynamique pendant un orgasme auto-induit par stimulation clitoridienne. Le premier cas était une femme incontinente à l'effort avec urgenturies sans hyperactivité détrusorienne sous-jacente. Une anomalie du col vésical était mise en évidence à l'urétrocystographie rétrograde et mictionnelle et une chute de la pression urétrale était observée au moment de l'orgasme, sans contraction du détrusor. Le deuxième cas était une femme incontinente (urgenturie, nycturie, pollakiurie) qui rapportait des fuites urinaires lors de la stimulation sexuelle intravaginale ou clitoridienne. Le bilan urodynamique mettait en évidence une hyperactivité détrusorienne associée à une dyssynergie vésicosphinctérienne. Pendant l'orgasme, on notait une augmentation de la pression endovésicale avec une baisse de la pression urétrale, accompagnées d'une fuite urinaire. Le troisième cas était une femme avec un antécédent de spina bifida. Elle présentait une énurésie depuis l'enfance. Sur une vessie hypoesthésique rétentionniste, et sans miction spontanée possible, on retrouvait une contraction du détrusor avec relaxation de l'urètre pendant l'orgasme. Chez les femmes présentant une hyperactivité détrusorienne, ni la sévérité des symptômes ni l'amplitude des contractions détrusoriennes n'était corrélé à la sévérité de l'incontinence coïtale.

El-Azab et al(10) ont considéré que l'incontinence pendant l'orgasme n'était pas uniquement en lien avec une hyperactivité détrusorienne, et qu'il existait une forte corrélation entre l'incontinence coïtale et la sévérité de l'incontinence à l'effort $(r=0.327, p=0.05)$, la parité et les prolapsus vaginaux. Dans cette étude, $90 \%$ des femmes incontinentes à l'effort avaient une incontinence pendant le coït. Moran et al(5) ont mis en avant la fréquence de l'incontinence urinaire à l'effort dans l'incontinence coïtale, quelle que soit sa forme. Ils ont conclu que l'incontinence durant les rapports sexuels entrait dans le cadre d'une incontinence à l'effort avec incompétence du sphincter urétral. Dans une étude observationnelle incluant 505 femmes sexuellement actives, Lau et al(8) ont montré que l'incontinence coïtale était en lien avec la sévérité de l'incontinence à l'effort et la diminution de la pression de clôture maximale $\left(\leq 30 \mathrm{cmH}_{2} \mathrm{O}\right)$, qui représentait le facteur prédictif significatif d'incontinence coïtale. Les auteurs ont rapporté quelques cas de femmes dont le bilan urodynamique était normal (20\% sur 281 femmes avec incontinence coïtale, $p=0,041)$. Pastor(14) a réalisé une revue de la littérature afin de différencier la fuite urinaire d'une éventuelle éjaculation, ou sécrétion de fluide au cours de l'orgasme. Il s'agissait de phénomènes bien distincts qui pouvaient être différenciés par une analyse biochimique des sécrétions. De plus, il concluait que l'incontinence coïtale était un symptôme causé par l'atteinte urétrale.

\section{Chez l'homme}

Chez l'homme, l'incontinence urinaire pendant les rapports sexuels s'observait principalement après prostatectomie. Celle-ci est connue pour avoir des répercussions directes sur la vessie, notamment un trouble de la compliance vésicale, et une incompétence sphinctérienne(4). Nombre sont les patients qui peuvent expérimenter des fuites urinaires pendant les rapports, c'était le cas de 20 à $64 \%$ des hommes en post-opératoire(15). Mitchell et al(16) ont montré que le taux d'incontinence coïtale diminuait avec le temps. Dans les 12 mois post-opératoire, en moyenne $24 \%$ présentaient une incontinence durant les rapports, alors qu'à plus d'un an de la chirurgie, le taux diminuait à $12 \%$ ( $\mathrm{p}<0,01)$. Parmi 1358 patients ayant eu une prostatectomie, $44,4 \%$ rapportaient être gênés de façon modérée à sévère par une incontinence pendant les rapports à 3 mois de l'intervention. Ce chiffre passait à $36,1 \%$ à 24 mois. Cette gêne était fortement associée à une incontinence à l'effort, mais les fuites pouvaient également apparaître en dehors d'un contexte d'incontinence à l'effort extracoïtale. Les fuites urinaires pendant les rapports survenaient majoritairement dans les 3 
premiers mois suivant la chirurgie et diminuaient avec le temps. Cependant, les auteurs n'ont pas pu définir à quelle phase du coït ces fuites se produisaient.

Le mécanisme étiopathogénique de l'incontinence coïtale, chez l'homme, serait une incontinence d'effort par insuffisance sphinctérienne secondaire soit à une lésion directe du sphincter urétral soit à une lésion de son innervation(17).

\section{Options thérapeutiques}

\section{Chez la femme}

La rééducation pelvi-périnéale a montré une amélioration de la fonction sexuelle chez les femmes ayant une incontinence à l'effort(18), notamment sur l'incontinence coïtale. En effet, au cours d'une étude randomisée, contrôlée, en simple aveugle, B $\emptyset$ et al ont mis en évidence une diminution de l'incontinence pendant les rapports ( 2 sur 20 versus 10 sur $25 ; \mathrm{RR}=0.25$, IC [0.06-1.01], $\mathrm{p}=0.02)(19)$. Une expérience avec une approche rééducative combinée sur 3 femmes, a montré que la combinaison de biofeedback, de stimulation électrique fonctionnelle, d'exercices musculaires du plancher pelvien et des cônes vaginaux améliorait les fuites urinaires pendant les rapports ainsi que la fonction sexuelle notamment le désir, l'excitation, la lubrification, l'orgasme, la satisfaction et la douleur(20).

Sur le plan pharmacologique, Serati et al(11) ont comparé l'efficacité d'un traitement antimuscarinique (tolterodine $4 \mathrm{mg}$ ) sur deux groupes femmes présentant une hyperactivité détrusorienne, dont l'un avec incontinence coïtale pendant l'orgasme. $41 \%$ des femmes qui avaient des fuites per-coïtales n'ont pas répondu au traitement (soit un taux d'efficacité de $59 \%)$, et $17 \%$ dans le groupe contrôle $(\mathrm{p}=0.023)$. L'efficacité des anticholinergiques était supérieur chez les femmes sans fuites durant les rapports (83\%). Ces résultats rejoignaient ceux de Sand(21) qui a montré une efficacité insuffisante des anticholinergiques (Oxybutinine) sur l'incontinence coïtale. Il n'y avait pas d'étude évaluant les béta3 agonistes adrénergiques de type mirabegron.

Sur le plan chirurgical, Ghezzi et al(22) ont évalué l'efficacité d'un traitement par bandelettes sous-urétrales dans l'incontinence coïtale, en incluant 53 femmes dans une étude prospective. Parmi elles, 43,4\% avaient expérimentés des fuites pendant les rapports, $91 \%$ pendant la pénétration et $9 \%$ pendant l'orgasme. Ils ont montré un taux de guérison de $98 \%$ pour l'incontinence urinaire d'effort et $87 \%$ pour l'incontinence coïtale. Dans une autre étude prospective, Pace and Vicentini(23) ont mis en évidence une efficacité de la bandelette transobturatrice avec un taux de guérison allant de 55 à $96 \%$ sur les fuites urinaires durant les rapports. Bekker et al ont réalisé une étude rétrospective chez 136 femmes pour évaluer les différentes bandelettes sous-urétrales. Ils ont mis en évidence une diminution du taux d'incontinence coïtale après chirurgie $(57 \%$ vs $12 \%, \mathrm{p}<0,001)(24)$. Dans une méta-analyse incluant 162 publications, Jha et al(25) ont rapporté une amélioration significative de l'incontinence coïtale après bandelettes ( $\mathrm{OR}=0,13$; IC [0,09-0,17]). Deux études ont montré que la colposuspension de Burch permettait un taux de guérison significatif ou une amélioration de l'incontinence coïtale, notamment avec une incontinence urinaire à l'effort associée(26,27). Baessler et Stanton avaient inclus 30 femmes dans une étude rétrospective. $73 \%$ d'entre elles avaient une incontinence coïtale à la pénétration, $10 \%$ à l'orgasme et $17 \%$ au deux moments. L'incontinence urinaire d'effort guérissait dans $77 \%$ des cas, et la colposuspension résolvait $70 \%$ des incontinences coïtales. L'hyperactivité détrusorienne n'était pas un critère d'exclusion.

\section{Chez l'homme}

Avant un rapport, il était conseillé comme chez la femme de vider la vessie. L'utilisation d'un préservatif pouvait éventuellement aider à surmonter la gêne occasionnée. La rééducation 
pelvi-périnéale a montré son efficacité dans l'incontinence coïtale masculine. Dans une étude randomisée contrôlée incluant 33 hommes sexuellement actifs après prostatectomie(28), la rééducation périnéale améliorait l'incontinence coïtale chez 6 sur 9 patients incontinents pendant les rapports contre 0 sur 8 dans le groupe contrôle $(\mathrm{p}=0,04)$. Une boucle pénienne de tension variable a également été testée et a montré des résultats positifs sur la diminution des fuites urinaires au cours des rapports(29). Quelques études ont testé un traitement pharmacologique par antidépresseur imipraminique(30) avec des résultats non probants. Quant au recours à la chirurgie, elle était possible qu'il s'agissait de la pose d'un sphincter artificiel ou de bandelettes sous urétrales(16).

\section{DISCUSSION}

Il n'était pas toujours facile de mettre en évidence une incontinence coïtale lors de l'interrogatoire, en raison de l'embarras des patients à rapporter de tels troubles même s'il s'avérait qu'ils en étaient le plus souvent très gênés. On notait ainsi une disparité dans les chiffres de prévalence de l'incontinence coïtale chez la femme. Cela pouvait être dû au fait que ce symptôme n'était pas recherché de la même façon selon les études (plaintes spontanées, auto-questionnaires, questions directes). Les questionnaires avaient souvent un taux de réponse plus élevé car ils permettaient d'éviter la consultation face à face et la gêne d'aborder une sphère d'intimité. Néanmoins, ces fuites devaient faire l'objet d'un interrogatoire précis afin d'en déterminer le type et le mécanisme. De plus, il convenait d'éliminer des diagnostics différentiels comme le diverticule urétral et la fistule vésicovaginale qui pouvaient s'exprimer par des fuites lors des rapports sexuels notamment lors de la pénétration ou des mouvements de va et vient. L'expulsion de sécrétions pendant les rapports sexuels n'était pas toujours bien définie, il était ainsi bien difficile, sans confirmation visuelle ou biochimique, de déterminer la nature du fluide expulsé pendant le rapport qu'il s'agissait d'un excès de lubrification vaginale, d'un écoulement utérin (hydrosalpinx) ou d'une perte d'urine. L'incontinence coïtale à la pénétration et celle pendant l'orgasme étaient deux entités distinctes sur le plan étiopathogénique. Il semblerait que les fuites urinaires à la pénétration relèveraient d'une incontinence à l'effort (si tant est que tout diverticule urétral soit éliminé) surtout si l'importance et la fréquence de ces fuites variaient en fonction de la position sexuelle de survenue, de l'intensité de l'activité sexuelle, et de l'absence d'urgence mictionnelle ressentie. Celles survenant pendant l'orgasme seraient plutôt corrélées à une hyperactivité détrusorienne surtout s'il existait simultanément une sensation d'urgenturie, mais ces fuites per-orgasmiques pouvaient également être rapprochées des autres incontinences aux paroxysmes émotionnels (frayeur, fou-rire) où l'on savait que l'instabilité urétrale pouvait aussi être incriminée.

Khan et al ont émis l'hypothèse que l'orgasme était un déclencheur de contraction non inhibée du détrusor et pouvait être associé à une inhibition de l'urètre causant sa relaxation. La fuite urinaire en serait la conséquence. La plupart des auteurs évoquait l'hypocompétence urétrale comme un mécanisme possible dans le cadre d'une incontinence coïtale. Ceci expliquerait l'échec des anti-muscariniques chez les patientes ayant une hyperactivité détrusorienne associée à une incontinence coïtale. De plus, Serati et al(7) rapportaient que les femmes avec hyperactivité détrusorienne et incontinence pendant l'orgasme présentaient une moins bonne réponse aux anti-muscariniques. Ils concluaient ainsi que l'incontinence pendant l'orgasme était un facteur de risque de résistance aux traitements anti-muscariniques. L'orgasme est un état émotionnel intense, tout comme le fou rire, la peur, l'angoisse. L'incontinence coïtale pourrait ainsi être rapprochée sur le plan physiopathologique de l'incontinence au fou rire («giggle incontinence »). Le méthylphénidate (Ritadine@) a montré son efficacité dans l'incontinence au fou rire, bien que son mécanisme d'action ne soit pas 
clair(31). Il n'a jamais été utilisé dans l'incontinence coïtale.

L'incontinence pendant la pénétration était liée à une hypermobilité urétrale(32). Celle-ci était la conséquence de l'intromission du pénis en érection, qui modifiait la position anatomique du col vésical, et associée à une augmentation de la pression abdominale entraînait une fuite urinaire. Dans une étude incluant 364 femmes, Thiede(33) a mis en évidence qu'une hyperpression abdominale par surpoids était corrélée à l'augmentation de la pression intravésicale, ce qui pouvait être à l'origine de fuites urinaires per-coïtales par défaut de transmission de la pression à l'urètre. En cas de prolapsus, il s'agissait d'un mécanisme purement mécanique, l'insertion du pénis pouvant réduire le prolapsus et démasquer une incontinence(34),(10). Par ailleurs, il n'y avait pas de différence morphologique à l'IRM au niveau du muscle releveur de l'anus chez les femmes incontinentes à l'effort avec ou sans incontinence coïtale.

En diminuant la fréquence des symptômes urinaires pendant les rapports sexuels, l'efficacité de la pose de bandelettes sous-urétrales apportait un argument sur le mécanisme étiopathogénique de l'incontinence coïtale par hypermobilité. Mais ces études ne prenaient en compte que les fuites urinaires pendant la pénétration et celles pendant l'orgasme, sans distinguer les autres formes d'incontinence coïtale.

Il existait d'autres facteurs physiopathologiques d'incontinence urinaire per-coïtale. Les femmes ayant une incontinence pendant l'orgasme pouvaient également avoir des fuites urinaires pendant la masturbation ou d'autres formes d'activité sexuelle. C'était le cas lors des atteintes médullaires. 51,7\% des femmes blessées médullaires avaient une incontinence urinaire pendant les rapports sexuels et cette incontinence pouvait survenir dès la stimulation du clitoris(35), (36). L'hypothèse physiopathologique serait l'activation d'un réflexe médullaire lors de cette stimulation périnéale locale induisant ainsi des contractions non inhibées du détrusor, au même titre que des percussions sus pubiennes ou d'autres stimulations cutanées locales (étirement de la marge anale) déclenchant chez les paraplégiques des mictions réflexes.

Restait enfin la dimension psycho-pathologique de l'incontinence urinaire per-coïtale. Ces fuites, si elles avaient réellement été présentes, étaient d'évidentes raisons d'abstention sexuelle et pouvaient d'ailleurs constituer une demande de prise en charge spécifique éventuellement chirurgicale tout à fait licite et acceptable. Mais ces fuites per-coïtales pouvaient parfois être imaginées (à côté d'authentiques fuites à l'effort extra-coïtales) et devenir ainsi un alibi pour l'évitement des rapports sexuels en cas de perte de la libido, de pathologies sexuelles associées (dyspareunie) ou de mésentente dans le couple.

Sur le plan thérapeutique, l'incontinence coïtale pendant la pénétration pouvait être traitée de façon chirurgicale dans près de $80 \%$ des cas. L'incontinence pendant l'orgasme pouvait être améliorée à $60 \%$ après un traitement pharmacologique adéquat. Des résultats probants ont été observés avec la rééducation pelvi-périnéale et la stimulation vaginale électrique apparaissait comme une thérapie adaptée(14).

Les possibles options thérapeutiques, en fonction du mécanisme étiopathologique de l'incontinence coïtale, sont répertoriées dans le tableau 1.

Chez l'homme le mécanisme n'a pas été complètement élucidé également, ce qui rendait difficile l'étude physiopathologique. Pendant le rapport sexuel, les sphincters urétraux interne et externe ainsi que le col vésical étaient fermés, créant une pression importante dans l'urètre prostatique. Au moment de l'orgasme, l'ouverture brutale du sphincter urétral externe, la haute pression intra prostatique associée à une contraction des muscles du périnée (ischiocaverneux et bulbospongieux) provoquait une éjaculation antégrade. Lors de l'éjaculation, la vessie se contractait de façon réflexe et une émission d'urine pouvait survenir si le patient présentait une insuffisance sphinctérienne(30). De plus, une lésion du nerf 
pudendal, qui innervait le sphincter urétral externe et le col vésical, pouvait être à l'origine de fuites urinaires pendant les rapports sexuels. Ce nerf avait un rôle dans la fonction sexuelle, il donnait naissance au nerf dorsal du pénis, qui véhiculait en partie la sensibilité sexuelle(4).

La possibilité d'une incontinence coïtale en post-prostatectomie pouvait être le fait de la technique chirurgicale utilisée bien qu'il n'y avait pas de différence démontrée sur la prévalence de l'incontinence coïtale après différentes techniques de prostatectomie. Cependant, cela avait un impact sur le temps de récupération(4), facteur à prendre en compte et dont il était important d'informer le patient avant l'intervention.

De façon plus générale, toute atteinte anatomique du plancher pelvien que ce soit après une chirurgie pelvienne ou sur une neuropathie d'étirement étaient des facteurs prédictifs d'incontinence à l'effort et d'incontinence coïtale. En effet, en causant une faiblesse du plancher pelvien associée à une atteinte du nerf pudendal, ces neuropathies entrainaient une diminution de la pression de clôture urétrale, une hyperactivité détrusorienne et une altération du sphincter externe. Cruz et al(37) ont montré sur des rats ayant eu une dénervation bilatérale du nerf pudendal, que $67 \%$ des femelles expulsaient des urines après l'éjaculation du mâle.

\section{CONCLUSION}

L'incontinence coïtale est un symptôme fréquent et gênant. Elle nécessite une analyse sémiologique précise (incontinence pendant la stimulation locale, l'excitation, la pénétration, les mouvements de va et vient, l'orgasme) permettant une présomption physiopathologique (hypermobilité cervico-urétrale, incompétence sphinctérienne, instabilité urétrale, hyperactivité détrusorienne) et une adaptation des stratégies thérapeutiques. C'est en règle l'interrogatoire et l'examen clinique qui permettent ces précisions, la place des examens complémentaires et notamment urodynamiques, n'étant pas actuellement clarifiée. Des thérapeutiques spécifiques qu'elles soient rééducatives, médicamenteuses (anticholinergiques) ou chirurgicales (bandelettes, sphincter) peuvent être proposées pour répondre aux demandes des patients.

Les auteurs déclarent ne pas avoir de liens d'intérêts. 


\begin{tabular}{|c|c|c|c|}
\hline $\begin{array}{l}\text { Caractéristiques de } \\
\text { l'incontinence coïtale }\end{array}$ & Type d'incontinence & Physiopathologie & Traitement \\
\hline $\begin{array}{l}\text { Stimulation clitoridienne et } \\
\text { caresses périnéales }\end{array}$ & Incontinence avec urgence & $\begin{array}{l}\text { Libération du réflexe } \\
\text { mictionnel : étiologie } \\
\text { neurologique fortement } \\
\text { suspectée ; hyperactivité } \\
\text { détrusorienne }\end{array}$ & $\begin{array}{l}\text { Anticholinergiques ; parfois } \\
\text { stimulation tibiale voire } \\
\text { Botox }\end{array}$ \\
\hline Dès la pénétration & Incontinence sans besoin & $\begin{array}{l}\text { Éliminer diverticule urétral } \\
\text { vérifier si prolapsus (effet } \\
\text { « réintégrateur » de la } \\
\text { pénétration pénienne avec } \\
\text { suppression de l'effet } \\
\text { pelote) }\end{array}$ & Traitement chirurgical \\
\hline Lors du va et vient & $\begin{array}{l}\text { Incontinence sans besoin } \\
\text { variable selon les positions } \\
\text { et l'intensité du rapport }\end{array}$ & $\begin{array}{l}\text { IUE « standard »: } \\
\text { hypermobilité cervico- } \\
\text { urétrale } \pm \text { insuffisance } \\
\text { sphinctérienne }\end{array}$ & $\begin{array}{l}\text { Rééducation ; traitement } \\
\text { chirurgical en cas d'échec } \\
\text { en fonction de la demande }\end{array}$ \\
\hline Lors de l'orgasme & $\begin{array}{l}\text { Incontinence souvent sur } \\
\text { urgence ; parfois insensible }\end{array}$ & $\begin{array}{l}\text { Instabilité urétrale ; } \\
\text { hyperactivité du détrusor }\end{array}$ & $\begin{array}{l}\text { Rééducation; } \\
\text { neurostimulation tibiale ; } \\
\text { alphabloquants ; } \\
\text { anticholinergiques ; beta3 }\end{array}$ \\
\hline
\end{tabular}

Tableau 1 : Sémiologie clinique des différentes formes d'incontinence coïtale, leurs mécanismes physiopathologiques et les options thérapeutiques possibles. 


\section{Références}

1. Zorzon M, Zivadinov R, Bosco A, Bragadin LM, Moretti R, Bonfigli L, et al. Sexual dysfunction in multiple sclerosis: a case-control study. I. Frequency and comparison of groups. Mult Scler. 1999;5(6):418- 27.

2. de Tayrac R, Haylen BT, Deffieux X, Hermieu JF, Wagner L, Amarenco G, et al. Traduction française de la terminologie commune International Urogynecological Association (IUGA)/International Continence Society (ICS) sur les troubles de la statique pelvienne chez la femme initialement publiée dans Int Urogynecol J 2010;21(1):5-26. Prog Urol. 2016;26(4):197- 225.

3. Hilton P. Urinary incontinence during sexual intercourse: a common, but rarely volunteered, symptom. Br J Obstet Gynaecol. 1988;95(4):377- 81.

4. Mendez MH, Sexton SJ, Lentz AC. Contemporary Review of Male and Female Climacturia and Urinary Leakage During Sexual Activities. Sex Med Rev. 2017.

5. Moran PA, Dwyer PL, Ziccone SP. Urinary leakage during coitus in women. J Obstet Gynaecol. 1999;19(3):286- 8.

6. Duralde ER, Rowen TS. Urinary Incontinence and Associated Female Sexual Dysfunction. Sex Med Rev. 2017;5(4):470- 85.

7. Serati M, Salvatore S, Uccella S, Nappi RE, Bolis P. Female Urinary Incontinence During Intercourse: A Review on an Understudied Problem for Women's Sexuality. J Sex Med. 2009;6(1):40- 8 .

8. Lau H-H, Huang W-C, Su T-H. Urinary leakage during sexual intercourse among women with incontinence: Incidence and risk factors. PLoS One. 2017;12(5).

9. Madhu C, Hashim H, Enki D, Yaasin M, Drake M. Coital Incontinence: What Can We Learn From Urodynamic Assessment? Urology. 2015;85(5):1034- 8.

10. El-Azab AS, Yousef HA, Seifeldein GS. Coital incontinence: Relation to detrusor overactivity and stress incontinence. Neurourol Urodyn. 2011;30(4):520- 4.

11. Serati M, Salvatore S, Uccella S, Cromi A, Khullar V, Cardozo L, et al. Urinary Incontinence at Orgasm: Relation to Detrusor Overactivity and Treatment Efficacy. Eur Urol. 2008;54(4):911- 7.

12. Vierhout ME, Gianotten WL. Mechanisms of urine loss during sexual activity. Eur J Obstet Gynecol Reprod Biol. 1993;52(1):45- 7.

13. Khan Z, Bhola A, Starer P. Urinary incontinence during orgasm. Urology. 1988;31(3):279- 82.

14. Pastor Z. Female ejaculation orgasm vs. coital incontinence: a systematic review. J Sex Med. 2013;10(7):1682- 91.

15. Fode M, Serefoglu EC, Albersen M, Sønksen J. Sexuality Following Radical Prostatectomy: Is Restoration of Erectile Function Enough? Sex Med Rev. 2017;5(1):110- 9.

16. Mitchell SA, Jain RK, Laze J, Lepor H. Post-Prostatectomy Incontinence During Sexual Activity: A Single Center Prevalence Study. J Urol. 2011;186(3):982- 5. 
17. Salomon L, Droupy S, Yiou R, Soulié M. Résultats fonctionnels et prise en charge des troubles fonctionnels après prostatectomie totale. Prog Urol. 2015;25(15):1028- 66.

18. Dumoulin C, Hay-Smith J. Pelvic floor muscle training versus no treatment for urinary incontinence in women. A Cochrane systematic review. Eur J Phys Rehabil Med. 2008;44(1):47- 63 .

19. Bø K, Talseth T, Vinsnes A. Randomized controlled trial on the effect of pelvic floor muscle training on quality of life and sexual problems in genuine stress incontinent women. Acta Obstet Gynecol Scand. 2000;79(7):598- 603.

20. Rivalta M, Sighinolfi MC, De Stefani S, Micali S, Mofferdin A, Grande M, et al. Biofeedback, electrical stimulation, pelvic floor muscle exercises, and vaginal cones: a combined rehabilitative approach for sexual dysfunction associated with urinary incontinence. J Sex Med. 2009;6(6):1674- 7.

21. Sand PK, Goldberg RP, Dmochowski RR, McIlwain M, Dahl NV. The impact of the overactive bladder syndrome on sexual function: a preliminary report from the Multicenter Assessment of Transdermal Therapy in Overactive Bladder with Oxybutynin trial. Am J Obstet Gynecol. 2006;195(6):1730- 5.

22. Ghezzi F, Serati M, Cromi A, Uccella S, Triacca P, Bolis P. Impact of tension-free vaginal tape on sexual function: results of a prospective study. Int Urogynecol J Pelvic Floor Dysfunct. 2006;17(1):54- 9 .

23. Pace G, Vicentini C. Female sexual function evaluation of the tension-free vaginal tape (TVT) and transobturator suburethral tape (TOT) incontinence surgery: results of a prospective study. $\mathrm{J}$ Sex Med. 2008;5(2):387- 93.

24. Bekker M, Beck J, Putter H, Venema P, à Nijeholt AL, Pelger R, et al. Sexual Function Improvement Following Surgery for Stress Incontinence: The Relevance of Coital Incontinence. J Sex Med. 2009;6(11):3208- 13.

25. Jha S, Ammenbal M, Metwally M. Impact of incontinence surgery on sexual function: a systematic review and meta-analysis. J Sex Med. 2012;9(1):34- 43.

26. Moran P, Dwyer PL, Ziccone SP. Burch colposuspension for the treatment of coital urinary leakage secondary to genuine stress incontinence. J Obstet Gynaecol. 1999;19(3):289- 91.

27. Baessler K, Stanton SL. Does Burch colposuspension cure coital incontinence? Am J Obstet Gynecol. 2004;190(4):1030- 3.

28. Geraerts I, Poppel HV, Devoogdt N, Groef AD, Fieuws S, Kampen MV. Pelvic floor muscle training for erectile dysfunction and climacturia 1 year after nerve sparing radical prostatectomy: a randomized controlled trial. Int J Impot Res. 2016;28(1):9.

29. Mehta A, Deveci S, Mulhall JP. Efficacy of a penile variable tension loop for improving climacturia after radical prostatectomy. BJU Int. 2013;111(3):500- 4.

30. Choi JM, Nelson CJ, Stasi J, Mulhall JP. Orgasm Associated Incontinence (Climacturia) Following Radical Pelvic Surgery: Rates of Occurrence and Predictors. J Urol. 2007;177(6):2223- 6.

31. Logan BL, Blais S. Giggle incontinence: Evolution of concept and treatment. J Pediatr Urol. 2017;13(5):430- 5 . 
32. Costantini E, Porena M, Giannitsas K, Athanasopoulos A, Balsamo R, Masiello G, et al. Coital incontinence: prevalence and risk factors in incontinent women. ics.org. 2016.

33. Thiede HA, Thiede FK. A glance at the urodynamic database. J Reprod Med. 1990;35(10):925- 31.

34. Amarenco G, Lacroix P, Delille M-A, Verollet D, Jousse M. Sexualité et incontinence urinaire de la femme. Pelv Perineol. 2010;5(2):97- 101.

35. Anderson KD, Borisoff JF, Johnson RD, Stiens SA, Elliott SL. Spinal cord injury influences psychogenic as well as physical components of female sexual ability. Spinal Cord. 2007;45(5):349- 59.

36. Perrouin-Verbe B, Courtois F, Charvier K, Giuliano F. [Sexuality of women with neurologic disorders]. Prog Urol. 2013;23(9):594- 600.

37. Cruz Y, Juárez R, Medel A, Corona-Quintanilla DL, Pacheco P, Juárez M. Coital Urinary Incontinence Induced by Impairment of the Dorsal Nerve of the Clitoris in Rats. J Urol. 2016;195(2):507- 14.

38. Oh S-J, Choo M-S, Kim HS, Kim JC, Lee JG, Yun JM, et al. Generic and disease-specific health-related quality of life in women with coital incontinence: a prospective, multicenter study. Gynecol Obstet Invest. 2008;65(1):62- 7.

39. Pons ME, Clota MP. Coital urinary incontinence: impact on quality of life as measured by the King's Health Questionnaire. Int Urogynecol J. 2008;19(5):621- 5.

40. Deutsch V, Reyal F, Vincens E, Kane A, Dalmont C, Madelenat P. Incontinence urinaire d'effort et bandelette sous-urétrale : implications sur la sexualité. Gynecol Obstet Fertil. 2008;36(4):417- 21.

41. Grzybowska ME, Wydra DG. Coital incontinence: a factor for deteriorated health-related quality of life and sexual function in women with urodynamic stress urinary incontinence. Int Urogynecol J. 2017;28(5):697- 704.

42. Shaw C. A Systematic Review of the Literature on the Prevalence of Sexual Impairment in Women with Urinary Incontinence and the Prevalence of Urinary Leakage during Sexual Activity. Eur Urol. 2002;42(5):432- 40.

43. Jha S, Strelley K, Radley S. Incontinence during intercourse: myths unravelled. Int Urogynecol J. 2012;23(5):633- 7.

44. Gray T, Li W, Campbell P, Jha S, Radley S. Evaluation of coital incontinence by electronic questionnaire: prevalence, associations and outcomes in women attending a urogynaecology clinic. Int Urogynecol J. 2017;1- 10.

45. Nilsson AE, Carlsson S, Johansson E, Jonsson MN, Adding C, Nyberg T, et al. Orgasm- Associated Urinary Incontinence and Sexual Life after Radical Prostatectomy. J Sex Med. 2011;8(9):2632- 9.

46. Giuseppe PG, Pace G, Vicentini C. Sexual function in women with urinary incontinence treated by pelvic floor transvaginal electrical stimulation. J Sex Med. 2007;4(3):702- 7. 